Cahiers de recherches médiévales

\title{
La réécriture ludique et distanciée de motifs lyriques dans la Prise d'Orange
}

Lydie Louison

\section{(2) OpenEdition \\ Journals}

Édition électronique

URL : https://journals.openedition.org/crm/5623

DOI : $10.4000 / \mathrm{crm} .5623$

ISSN : 1955-2424

Éditeur

Honoré Champion

Édition imprimée

Date de publication : 20 juin 2008

Pagination : 99-111

ISSN : $1272-9752$

Référence électronique

Lydie Louison, "La réécriture ludique et distanciée de motifs lyriques dans la Prise d'Orange », Cahiers de recherches médiévales [En ligne], 15 | 2008, mis en ligne le 20 juin 2011, consulté le 15 décembre 2022. URL : http://journals.openedition.org/crm/5623 ; DOI : https://doi.org/10.4000/crm.5623 


\section{酶RM}

\section{La réécriture ludique et distanciée de motifs lyriques dans la Prise d'Orange}

En hommage et remerciement à C. Lachet, mon maître.

Abstract: Omnipresent in the Prise d'Orange, the reverdie's lyric motive contributes to a ludic rewriting of the epic. But this motive becomes also itself the target of a parody proceeding from burst, inversion and transfer of his various components.

Résumé: Omniprésent dans la Prise d'Orange, le motif lyrique de la reverdie contribue à une réécriture ludique de l'épopée. Mais ce motif en lui-même devient la cible de la parodie, procédant de l'éclatement, de l'inversion et du transfert de ses diverses composantes.

S'ouvrant sur une captatio benevolentiae apparemment conventionnelle, didactique et morale, la Prise d'Orange' s'annonce comme une bone chanson, un récit édifiant et bénéfique, que son prologue situe dans un horizon d'attente épique ${ }^{2}$. Toutefois, par sa polysémie, l'adjectif bone évoque aussi la qualité esthétique de la chanson, et l'intervention immédiate et martelée du je énonciateur annonce une œuvre originale, dont l'auteur revendique puissamment la paternité. Quelques indices disséminés dans les vers liminaires permettent de mieux définir cette originalité. La présentation d'Orable distingue en effet d'emblée l'héroïne des princesses sarrasines ${ }^{3}$ : loin de jouer un rôle épisodique, elle intervient dans plus de la moitié des laisses, elle est reine, mariée, et possède plutôt le profil d'une dame courtoise inaccessible, susceptible de susciter en Guillaume un amour-souffrance typiquement courtois ${ }^{4}$. S'il est maintenant admis, depuis la captivante démonstration de C. Lachet, que la Prise d'Orange, cette brillante "parodie courtoise d'une épopée », n'a rien d'un "plat recueil de lieux communs épiques », et que «la parodie, provoquée surtout par l'intrusion de la courtoisie, [...] prouve non

${ }^{1}$ La Prise d'Orange, chanson de geste de la fin du XII ${ }^{e}$ siècle, éd. C. Régnier, Paris, Klincksieck, $7^{\mathrm{e}}$ éd., 1986.

${ }^{2}$ À l'appel au public remplissant sa fonction phatique succède la promotion de l'œuvre, la valorisation du héros, Guillelme Fierebrace, personnage central d'un cycle épique, l'exaltation de la religion chrétienne, la mention du lignage, l'allusion au Charroi de Nîmes, ainsi que le résumé de l'intrigue notifiant la victoire des chrétiens sur les païens, la conversion et le baptême d'Orable.

${ }^{3}$ Le motif de la Sarrasine amoureuse d'un chevalier chrétien est bien étudié par C. Lachet dans La Prise d'Orange ou la parodie courtoise d'une épopée, Genève, Paris, Slatkine, 1986, p. 79-119.

${ }^{4}$ Prise d'Orange, v. 36-38.

${ }^{5}$ L. Gautier, Les Épopées françaises, Paris, Palmé, $2^{\mathrm{e}}$ éd., 1882, t. IV, p. 394.

Cahiers de Recherches Médiévales, 15, 2008 
seulement la lucidité de l'auteur sur l'automatisme des 'topoi' et des expressions figées dont usent et abusent les poètes, mais encore une parfaite connaissance de la littérature de son temps ${ }^{6}$, doit-on penser pour autant que le trouvère «transforme en partie la chanson de geste en une "canso' d'amour »? ? Déchu de son trône épique, devenu «Guillelme l'Amïable »", Fierebrace n'est pas réhabilité dans une autre échelle de valeurs, et la courtoisie insérée dans ces décasyllabes n'échappe pas au regard démythificateur et parodique du trouvère'. Poursuivant l'analyse lumineuse de $\mathrm{C}$. Lachet, nous ajouterons quelques pierres à son édifice en revenant sur la réécriture distanciée et ludique du modèle ${ }^{10}$ lyrique de la reverdie, qui sillonne toute la Prise d'Orange.

Particulièrement appréciée pour sa richesse symbolique, la reverdie présente un cadre récurrent en trois étapes ${ }^{11}$ : le poète sort à l'aube pour profiter des charmes $\mathrm{du}$ renouveau printanier et entre dans un bois; une rencontre amoureuse a lieu et occasionne un monologue ou un dialogue sentimental; la splendide jeune fille est

${ }^{6}$ C. Lachet, op. cit., p. 224-225.

${ }^{7}$ Ibid., p. 228 .

${ }^{8}$ Prise d'Orange, v. 1563.

${ }^{9}$ Cet aspect est évoqué par C. Lachet, op. cit., p. 216-217.

${ }^{10}$ Nous reprenons cette notion à P. Zumthor, «Intertextualité et mouvance », Littérature, 1981, février, n41, p. 8-16. Le poète de la Prise d'Orange ne joue pas en effet avec un texte en particulier, mais avec les constantes d'un genre lyrique. Or dans « Ironie, satire, parodie. Une approche pragmatique de l'ironie », Poétique, 1981, p. 140-155, L. Hutcheon rappelle que «la parodie ne peut avoir pour cible qu'un texte ou des conventions littéraires. » Nous entendrons donc par parodie la réécriture ludique et décalée d'un hypotexte, d'un modèle, d'un thème, d'un motif ou d'une formule stéréotypés nettement identifiables, dans le but de faire rire ou sourire le lecteur. Sur ce type d'écriture, voir par exemple M.A. Rose, Parody/Meta-fiction. An analysis of Parody as a critical Mirror of the Writing and the Reception of Fiction, Londres, Croom Helm, 1979; G. Genette, Palimpsestes. La littérature au second degré, Paris, Le Seuil, 1982 ; Le singe à la porte. Vers une théorie de la parodie, GROUPAR éd., New York, Peter Lang, 1984 ; L. Hutcheon, A Theory of Parody. The Teaching of Twentieth Century Art Forms, New York, London, Pethuen, 1985 ; M. Hannoosh, Parody and decadence. Laforgue's Moralités legendaries, Colombus, Ohio State University Press, 1989 ; D. Sangsue, La Parodie, Paris, Hachette, 1994 ; P. Eichel-Lojkine, Excentricité et humanisme: parodie, dérision et détournement des codes à la Renaissance, Genève, Droz, 2002.

${ }^{11}$ En ce qui concerne la typologie de la reverdie, voir M. Picarel, «Le début printanier dans les chansons des troubadours Marcabru et Bernart de Ventadour», Annales de l'Institut d'Études Occitanes, II, 1970, p. 169-190 ; M. Tyssens, «En avril au tens pascour », Mélanges de philologie romane dédiés à la mémoire de Jean Boutière (1899-1967), Liège, 1971, I, p. 589-603, P. Bec, La Lyrique française au Moyen Âge (XII ${ }^{e}-X I I I^{e}$ siècles), Paris, Picard, 1977 , t. 1, p. 136-141, t. 2, p. 58-63 ; M. Le Person, "L'insertion de la 'reverdie' comme ouverture ou relance narrative dans quelques romans des $\mathrm{XII}^{\mathrm{e}}$ et $\mathrm{XIII}{ }^{\mathrm{e}}$ siècles », Les Genres insérés dans le roman, Actes du colloque International du 10 au 12 décembre 1992, Université Jean Moulin Lyon III, C.E.D.I.C., 1993, p. 17-33 ; F. Vigneron, Les Saisons dans la poésie française des $X I V^{e}$ et $X V^{e}$ siècles, Paris, Champion, 2002. 
décrite. Ce genre lyrique présente des contours juste assez nets et connus pour susciter, dès la fin du XII ${ }^{\mathrm{e}}$ siècle, des réécritures romanesques ou épiques ${ }^{12}$, donner naissance à une thématique stéréotypée, et stimuler, éventuellement, un esprit facétieux. Or dès le vers d'intonation de la laisse III, l'auteur de la Prise d'Orange semble s'appuyer sur l'adaptation épique du motif printanier, représentant le retour de la belle saison en des termes inspirés du Charroi de Nîmes : «Ce fu en mai, el novel tens d'esté $\rangle^{13}$. Il associe donc fermement deux matières, épique et lyrique, et constitue une mise en abyme de l'esthétique même de l'œuvre. Les vers suivants abandonnent toutefois l'épopée qui venait tout juste de commencer pour se consacrer à la reverdie, actualisant quelques-uns de ses éléments traditionnels ${ }^{14}$ : le mois de mai et le retour de la belle saison ${ }^{15}$, la verdure des prés et le fleurissement des bois $^{16}$, les ruisseaux qui retrouvent leur lit, le chant des oiseaux ${ }^{17}$ et le lever matinal du protagoniste ${ }^{18}$ :

12 Dans «Une ville devenue désir: La Prise d'Orange et la transformation du motif printanier », Mélanges de langue et de littérature médiévales offerts à Alice Planche, Nice, 1984, p. 21-32, N. Andrieux rappelle en effet qu'il existe une "actualisation du printemps commune, semble-t-il, à l'ensemble des textes dits courtois», et qu' «il en est une autre, propre à la chanson de geste ». Dans son étude, cette dernière se réduit à l'expression du renouveau et de la douceur, motif transitif favorisant la prise de conscience d'un manque et introduisant l'événement destiné à le combler. Elle reconnaît cependant que les chansons de geste peuvent développer parfois d'autres formes de la reverdie.

${ }^{13}$ Le Charroi de Nîmes, éd. bilingue C. Lachet, Paris, Gallimard, 1999, v. 14.

${ }^{14}$ Voir l'analyse de ce motif par C. Lachet, op. cit., p. 153-155. Si, comme le pense N. Andrieux, art. cit., ces éléments relèvent de la forme épique du topos, ces vers ne présentent aucune perspective ludique. Mais peut-on considérer que l'emprunt lyrique était déjà perçu comme un motif épique à la fin du XII ${ }^{\mathrm{e}}$ siècle, alors que la majorité des exemples cités par N. Andrieux sont postérieurs ou contemporains à la Prise d'Orange ? Dans la mesure où l'auteur de la Prise d'Orange s'inspire du motif lyrique dans sa globalité, rencontre amoureuse et description de la jeune fille incluses, nous considérerons qu'il comptait sur leur discordance au sein de l'épopée pour mieux asseoir ses jeux humoristiques. Peut-être le succès de la Prise d'Orange, appréciable au nombre des manuscrits qui nous sont parvenus, at-il participé à la cristallisation de ce motif sous sa forme épique, dans la mesure où le topos y est exceptionnellement récurrent, de l'ouverture au dénouement. C'est ce que confirme D. Boutet dans La Chanson de geste, Paris, PUF, 1993, p. 183-184 : «Ce motif paraît avoir essaimé dans le genre entier à partir de la geste de Guillaume (entendue au sens large), dont la tonalité méridionale et la thématique souvent courtoise étaient propres à accueillir cet héritage lyrique».

${ }^{15}$ Cf. Colin Muset, «En mai, quant li rossignolez», Les Chansons de Colin Muset, éd. J. Bédier, Paris, Champion, 1969, v. 1 ; «Volez oïr la muse Muset? / En mai fut fete un matinet », ibid., v. 1-2; «En mai au douz tens nouvel», Poèmes d'amour des XII et XII siècles, éd. E. Baumgartner et F. Ferrand, Paris, 10/18, 1983, p. 264, v. 1 ; « En avril au tens pascour », ibid., p. 260, v. 1.

${ }^{16} C f$. «En avril au tens pascour / Que seur l'erbe nest la flor », v. 1-2; «En mai au douz tens nouvel / Que raverdissent prael », v. 1-2; «Au tans novel que naissent flours », P. Bec, op. cit., n`51, v. 1 ; et Colin Muset, «Volez oïr la muse Muset?», v. 3 : «En un vergier flori, verdet $»$. 
Ce fú en mai, el novel tens d'esté ;

Florissent bois et verdissent cill pré,

Cés douces evves retraient en canel,

Cil oisel chantent doucement et soëf.

(La Prise d'Orange, v. 39-42)

Le processus même du renouveau végétal est rendu sensible par l'emploi conventionnel de verbes inchoatifs ${ }^{19}$, dont la longueur mime la lenteur de l'épanouissement floral. Du parallélisme syntaxique émane une poésie harmonieuse et équilibrée. La douceur du climat est suggérée par les allitérations en consonnes liquides, sifflantes et nasales, alors que les assonances en voyelles palatales soulignées ci-dessus annoncent le chant des oiseaux. Initialement implicite, la douceur ${ }^{20}$ devient explicite et envahit les vers 41 et 42 , bénéficie de caractérisations hyperboliques et s'amplifie rythmiquement dans une dérivation lexicale de l'adjectif «douces » à l'adverbe «doucement». Le présent de l'indicatif et les démonstratifs de notoriété désignent le topos tout en actualisant cette scène animée par les mouvements d'une nature débordante de vie. Développant une reverdie à peine esquissée dans le Charroi de Nîmes, l'enrichissant de détails puisés au cœur du modèle lyrique qu'il pastiche, le poète semble presque abandonner l'épopée pour confronter dans un but ludique deux univers littéraires aux horizons d'attente divergents, l'art lyrique et l'art épique. Par cette expansion poétique inattendue, il ménage un effet de surprise qu'il s'apprête à renouveler sans cesse. De fait, choisir la reverdie pour structurer une partie des jeux parodiques à l'œuvre dans la Prise d'Orange est éminemment significatif et pertinent: comment mieux suggérer le renouvellement profond, enjoué et enthousiaste de l'épopée, si ce n'est par la réécriture de ce genre lyrique symbolisant la réviviscence, la joie de vivre, l'exubérance, motif susceptible de devenir à son tour la cible d'une distanciation ludique?

Conformément au motif du renouveau printanier, Guillaume se lève en effet à l'aube, mais des décalages provoquent le sourire :

Li cuens Guillelmes s'est par matin levez,

Au moustier vet le servise escouter,

Puis s'en issi quant il fu definez

Et monta el palés Otran le deffaé,

\footnotetext{
${ }^{17} C f$. Colin Muset, «En mai, quant li rossignolez », v. 1 ; «Volez oïr la muse Muset ? », v. 5$6:$ : Ou chantoient cil oiselet / Par grant baudor»; «En avril au tens pascour », v. 3-4 : «L'aloete au point du jour/ Chante par mult grant baudor», v. 7-8: «S'oï chanter sur l'arbroisel / Un oiselet en son latin » et v. 11-21; Guillaume le Vinier, «Mout a mon cuer esjoï », P. Bec, op. cit., n`53, v. 1-11, «En mai au douz tens nouvel», v. 3-4, 11, 24, 29-32.

${ }^{18} C f$. «En avril au tens pascour », v. 6 : «Si me levai par un matin»; Colin Muset, «Volez oïr la muse Muset? », v. 2, $4:$ : En mai fut fete, un matinet, / [...] Au point du jour »; « Au tans novel que naissent flours », v. 4-5.

${ }^{19} C f$. « En mai au douz tens nouvel / Que raverdissent prael », v. 1-2.

${ }^{20} C f$. « En avril au tens pascour », v. 5 : « Pour la douçor du tens nouvel ».
} 
Qu'il ot conquis par sa ruiste fierté.

(La Prise d'Orange, v. 43-47)

Le vers 43 opère en effet un brutal retour à l'art épique en projetant « li cuens Guillelmes », personnage typique des chansons de geste, dans l'univers lyrique précédemment évoqué. Cadrée dans un hémistiche très stéréotypé, la dénomination du héros crée un contraste net et renvoie le lecteur-spectateur à l'horizon d'attente initial. Fierebrace se rend en outre à l'office liturgique, se pliant à la lettre au motif épique bien connu des dévotions matinales ${ }^{21}$. Son indifférence initiale pour le renouveau printanier interrompt brutalement le motif lyrique, accentue l'incompatibilité des deux univers et amorce un jeu parodique. Manifestement, Guillaume n'est pas encore un personnage courtois; il n'en est pas pour autant présenté comme un véritable héros épique. Le texte s'attarde en effet sur ses occupations ordinaires et exprime implicitement son ennui. L'inaction du héros transparaît dans la narration de gestes quotidiens - déplacements ou postures relatés d'abord au passé composé, puis au présent de narration et enfin au passé simple, temps de la rupture et de l'héroïsme, alors que les hauts-faits sont évoqués au passé antérieur, temps doublement révolu. Le schéma ascendant en gradation semble laudatif, Guillaume domine un palais qu'il a arraché aux mains des Sarrasins, mais le panorama épique décrit contraste puissamment avec le topos attendu et avec les exploits passés :

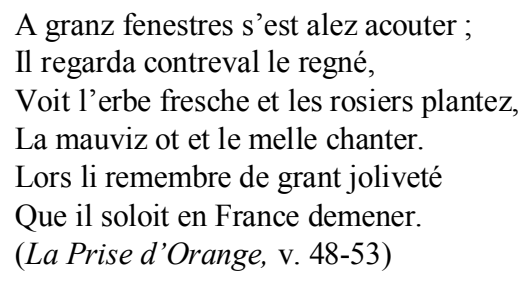

Dans une assonance antithétique, le verbe «acouter» indique une posture statique et passive en rupture avec la «ruiste fierté » du chevalier épique, et les vers à connotation lyrique multiplient les distorsions, provoquant des effets déceptifs et humoristiques. Au lieu de contempler «l'approche de l'invasion sarrasine, la terre ravagée, les incendies, les massacres ou la venue d'un messager, ou l'arrivée des secours espérés $»^{22}$, Guillaume «voit l'erbe fresche et les rosiers plantez»! Loin d'entendre le fracas des armes, le martèlement des sabots ou des cris de ralliement, il se laisse bercer par le chant des oiseaux et la « ruiste fierté » traditionnelle du héros ${ }^{23}$

${ }^{21}$ Concernant les motifs épiques, voir J. Rychner, La Chanson de geste, essai sur l'art épique des jongleurs, Genève, Droz, 1955, p. 126-139 et J.-P. Martin, Les Motifs dans la chanson de geste. Définition et utilisation, thèse universitaire publiée à l'Université de Lille III, 1992.

${ }_{22}^{2}$ J. Frappier, Les Chansons de geste du cycle de Guillaume d'Orange, Paris, SEDES, 1955, t. I, p. 112.

${ }^{23}$ L'auteur renvoie certainement au Couronnement de Louis. 
a cédé le pas devant la nostalgie d'une vie de plaisir ${ }^{24}$. La parodie procède ainsi par rupture de l'horizon d'attente épique et ingérence d'éléments exogènes inattendus tirés de la reverdie. Tendant à faire oublier le contexte épique, ces vers placent en outre le lecteur dans l'attente d'un chant lyrique et nostalgique, en écho au ramage des oiseaux ${ }^{25}$; or le jongleur propose une nouvelle rupture souriante. L'interpellation de Bertrand rappelle l'importance du lignage dans les chansons de geste, et fait de nouveau basculer le texte dans le domaine épique. Guillaume sort alors brutalement de sa léthargie pour justifier sa nostalgie, son ennui et son oisivetée ${ }^{26}$. Alors même que des topoi courtois ont envahi des vers épiques au risque de remettre en cause l'unité de l'œuvre, le héros regrette précisément l'absence de toute courtoisie dans sa vie, le manque de jongleurs, de plaisirs, de divertissements, de femmes, avant de déplorer l'absence d'ennemis et de combats susceptibles de le distraire et de lui permettre de faire la preuve de sa vaillance ${ }^{27}$. Ces préoccupations essentiellement courtoises provoquent encore, par ce décalage, un effet de surprise. Si l'écriture épique est ainsi parodiée par inversion ${ }^{28}$, par toutes les intrusions courtoises et par les aspirations mêmes du héros, la reverdie n'est pas épargnée: dupliquée dans les laisses similaires III et IV, elle subit tout d'abord un éclatement, les vers qui lui sont consacrés alternant avec la mention des dévotions matinales et celle d'un panorama censé être épique ${ }^{29}$.

Le motif est en outre réitéré, d'une part dans le récit initial de Guillebert, et d'autre part lorsque Guillaume et Guïelin pénètrent dans la cité d'Orange. Très éloignée du compte rendu militaire, la présentation d'Orange par le prisonnier s'ouvre de manière insolite :

S'i estiez le premier jor d'esté,

Lors orrïez les oseillons chanter,

Crïer faucons et ces ostoirs müez,

\footnotetext{
${ }^{24}$ Le profond sentiment de nostalgie est parfaitement suggéré à travers l'intériorisation progressive du héros, que révèle un regard dirigé vers les profondeurs du paysage, projection métonymique des profondeurs de l'âme. La synesthésie combinant la vue et l'ouïe suscite spontanément une réminiscence, qui s'impose à l'esprit de Guillaume, toujours plus passif. Si, au début du XIII ${ }^{\mathrm{e}}$ siècle, les Enfances Vivien (éd. C. Wahlund et H. von Feilitzen, Uppsala, Paris, 1895), v. 911-918 et 926-928, proposent quelques vers similaires, la nostalgie y est profondément épique, car Vivien pleure l'absence de membres de sa famille.

${ }^{25}$ Prise d'Orange, v. 51.

${ }^{26}$ C'est une valeur courtoise essentielle, car l'oisiveté rend disponible au «deduit» et à l'amour, dont Guillaume peut alors ressentir le manque. Cette entrée en matière annonce le rôle d'Oiseuse dans le Roman de la Rose de Guillaume de Lorris.

${ }^{27}$ Prise d'Orange, v. 54-69 et 85-100.

${ }^{28}$ Déplorer l'absence d'ennemis alors qu'on possède en abondance des vivres et des armes renverse les topoi bien connus de la présence d'ennemis en surnombre et de la pénurie. En outre, un véritable héros épique n'attend pas que l'ennemi attaque pour prendre les armes. Cette inversion parodique est encore soulignée par la mauvaise foi de Guillaume et met en évidence sa passivité.

${ }^{29} \mathrm{La}$ parodie du panorama épique procède également par éclatement, l'arrivée du messager étant repoussée jusqu'au vers 110 par les plaintes de Guillaume.
} 
Chevaux hennir et cez muls rechaner,

Ces Sarrazins deduire et deporter.

(La Prise d'Orange, v. 245-249)

Comment ne pas sourire du style élevé dans lequel Guillebert exprime sa fascination pour une ville sarrasine qu'il devrait haïr ou vouloir conquérir? Et depuis quand un prisonnier maltraité, fraîchement échappé et épuisé réalise-t-il l'éloge de sa geôle en introduisant des hémistiches lyriques par des formules d'actualisation épiques? La situation incongrue met en évidence la réécriture distanciée de la reverdie, placée pour l'occasion dans le discours d'un personnage sale, maigre et décharné. Cette parodie s'inaugure en outre dans un jeu finement caricatural et signalétique, la situation temporelle excessivement précise «le premier jor d'esté » désignant en effet le motif visé. Ce dernier subit ensuite une dépoétisation parodique : contaminée par les topoi de l'entrée dans la ville et de la peinture de la cité radieuse ${ }^{30}$, la reverdie est privée de sa délicate poésie et envahie, dans une gradation dysphorique, par des animaux et des Sarrasins indénombrables et de plus en plus bruyants, dont les cris cacophoniques finissent par anéantir l'harmonieux chant des oiseaux. La transposition ludique est encore accentuée par une connotation sensuelle inhabituelle, inversant le symbolisme traditionnel de l'aube. Si les «faucons» et «ostoirs»- animaux nobles relevant d'un monde courtois et raffiné pratiquant la chasse - symbolisent « la victoire du principe mâle, diurne, solaire $»^{31}$, les chevaux connotent "l'impétuosité du désir» et les mulets représentent la puissance sexuelle masculine sous sa forme primitive. Du passage émane une atmosphère sensuelle $\mathrm{e}^{32}$ : inhabituels pour caractériser les païens, les infinitifs «deduire et deporter $»^{33}$ renversent d'une part l'hémistiche épique traditionnel « et glatir et uller », évoquent des divertissements courtois, et connotent d'autre part un vaste champ de plaisirs, dont les épices, qui se substituent au renouveau floral, accentuent la suavité ${ }^{34}$. L'inversion humoristique d'un motif lyrique symbolisant la pureté semble alors légitimée par la subjectivité hédoniste des propos de Guillebert. Ce dernier s'avère si fasciné par la cité dont il s'est échappé et par les plaisirs auxquels il n'a pu goûter qu'il en oublie son emprisonnement, excite involontairement la convoitise de Fierebrace en faisant miroiter sous ses yeux l'objet encore virtuel de ses désirs, et met en place une situation piquante. Or le vécu de Guillaume corroborant la description de Guillebert, la similitude des vers 245-252 et 409-414 et de leur assonance engendre un savoureux comique de répétition. L'écho

\footnotetext{
${ }^{30}$ Les procédés transformationnels analysés par N. Andrieux, art. cit., p. 28 (addition d'animaux, substitution de la perception olfactive d'épices à celle, visuelle, du renouveau floral, et effacement des expressions de la douceur et des végétaux) s'avèrent parodiques.

${ }^{31}$ Voir J. Chevalir, A. Gheerbrant, Dictionnaire des symboles, Paris, Robert Laffont, 1982.

${ }^{32}$ Cette atmosphère est largement appuyée par les synesthésies, Guillebert sollicitant d'abord l'ouïe, puis l'odorat et la vue de ses interlocuteurs. Par la suite, il fait même appel au toucher lorsqu'il décrit le corps svelte d'Orable (v. 256, 278).

${ }^{33}$ Le verbe «deporter» est d'ailleurs repris à l'assonance du vers 260 pour évoquer les plaisirs qu' " uns gentils hom » est susceptible de goûter auprès d'Orable.

${ }^{34}$ Pour un traitement différent du motif, voir par exemple Floire et Blanchefleur, v. 20352040.
} 
épique ainsi singé est envahi par un motif courtois, dont la réécriture distanciée est réitérée. La parodie par réduplication et diffraction du motif lyrique atteint en outre une dimension structurelle : la description de la jeune femme réalisée par Guillebert ${ }^{35}$ est en effet reprise à partir du vers 660 lors de la rencontre amoureuse, et les aveux sont retardés jusqu'au vers 1376.

L'unité du motif est toutefois soulignée par le cadre enchanteur dans lequel se déroule cette rencontre : la description du jardin intérieur de Gloriette proroge la reverdie et en corrobore la réécriture parodique par éclatement. L'auteur s'amuse en outre à retarder encore l'apparition d'Orable, tout en l'annonçant sans cesse : relatant la progression des Chrétiens dans Orange et dans Gloriette, il prend le temps de décrire la tour et ses charmes ${ }^{36}$, concluant sur un vers ambigu qui pourrait qualifier Orable et lancer son portrait: «Bien fu paree, mout par fu avenant $»^{37}$. La phrase suivante poursuit ce quiproquo, différant le sujet par un déploiement d'éléments spatiaux: «A une part de la chambre leanz / Avoit un pin [...]». Mais partageant l'impatience du héros, quel sujet peut attendre le lecteur avant la césure du vers 652 , sinon Orable si savamment annoncée? La chute n'en est que plus amusante, car cet arbre épique notoire ${ }^{38}$, dont l'auteur développe la description après une intervention ironique, présente des qualités féminines typiquement courtoises accentuées dans un chiasme, image de la perfection ${ }^{39}$ : des branches aussi longues que peuvent l'être les bras des belles dames, des feuilles aussi grandes que des doigts délicats. Quant à la fleur, blanche et vermeille, elle possède les deux couleurs caractéristiques de la beauté féminine médiévale. Non seulement la description du pin vient surseoir celle d'Orable, mais elle est en outre émaillée d'éléments annonçant la reine, ce qui ne peut qu'attiser l'impatience du lecteur, gagné par le point de vue subjectif et passionné de Guillaume. Avec humour, la rencontre amoureuse tant attendue est encore différée par une allusion aux fréquentes entrevues qui se déroulent dans ces lieux et par l'évocation d'épices odorantes et sensuelles, confirmant l'authenticité des propos de Guillebert ${ }^{40}$.

Accentué par ces jeux déceptifs, le fin jeu parodique ne repose toutefois pas seulement sur l'éclatement des motifs. Il procède encore et toujours de l'association d'éléments incompatibles: ainsi la reverdie célébrant le renouveau printanier se déploie-t-elle finalement à rebours à travers un jardin intérieur clos, abrité du soleil et du vent, plus proche des jardins exotiques de Babylone; les oiseaux et leurs trilles ont disparu, remplacés par le silencieux aigle d'or; lorsqu'elles ne sont pas «painte a or et par mestrie $»^{41}$, les fleurs et les feuilles s'épanouissent sur un pin, arbre épique

\footnotetext{
${ }^{35}$ La première description d'Orable est faite par Guillebert, v. 202-207, 252-259, 275-282.

${ }^{36} \mathrm{La}$ parodie procède alors par répétition, contrefaisant le principe même des échos épiques avec humour puisque la description de Gloriette est similaire à celle de l'entrée dans Orange. Les vers 646-649 reprennent les vers 460-463.

${ }^{37}$ Prise d'Orange, v. 650.

${ }^{38}$ Depuis La Chanson de Roland, éd. J. Dufournet, Paris, GF-Flammarion, 1993, v. 114, 165, $168,500,2357,2375$.

${ }^{39}$ "Longue est la branche et la fueille en est grant», Prise d'Orange, v. 654.

${ }^{40}$ Prise d'Orange, v. 250-251.

${ }^{41}$ Prise d'Orange, v. 274.
} 
par excellence, conifère aux aiguilles persistantes pourtant incongru pour signifier le renouveau végétal; au parfum dégagé par la flore renaissante se substitue celui des épices. Si ces quelques fils lyriques relevant de la reverdie semblent ainsi s'intégrer à la trame épique, leur discordance ne peut manquer de susciter le sourire, et l'auteur renverse le procédé: à la princesse sarrasine finalement attendue dans un tel contexte succède la « dame d'Aufriquant », sensuelle, certes, mais d'une splendeur répondant aux canons de la beauté féminine occidentale et faisant d'elle une dame courtoise $^{42}$. L'auteur sollicite ainsi la culture des lecteurs et fait surgir avec brio un univers et des personnages complexes, d'origine tantôt épique, tantôt courtoise, tirant toujours un domaine vers l'autre, mêlant constamment les deux écritures afin qu'elles dénoncent réciproquement leurs automatismes dans un jeu purement littéraire à double tranchant.

La descriptio puellae $e^{43}$ - exercice de style courtois s'il en est, au cours duquel un auteur peut faire la preuve de son art en parant à sa manière un modèle légué par la tradition - est ainsi également parodiée par un refus de l'ornementation et par suppression. L'auteur de la Prise d'Orange n'a en effet conservé qu'un trait conventionnel qu'il accentue de surcroît par deux comparaisons hyperboliques: «Ele est plus blanche que la noif qui resplent / et plus vermeille que la rose flerant $»^{44}$. Le jeu caricatural consiste tout d'abord à dépouiller le stéréotype, à séparer ensuite les deux teintes fondant l'harmonieux contraste chromatique traditionnel ${ }^{45}$, à les amplifier à la personne entière jusqu'à ce que ce contraste devienne plaisant, le parallélisme des comparaisons appuyant la dimension mécanique et systématique de la rhétorique courtoise. La réécriture parodique par grossissement est d'autant plus savoureuse qu'elle est préparée en amont par une onomastique choisie en gradation pour son homophonie avec l'isotopie de la couleur

\footnotetext{
${ }^{42}$ Orable se situe toujours à mi-chemin entre la Sarrasine amoureuse et la dame courtoise, ce qui permet à l'auteur de jouer avec les deux motifs tirés respectivement de l'écriture épique et de l'esthétique courtoise.

${ }^{43}$ À ce sujet, voir par exemple A. Colby, The Portrait in Twelfth Century French Literature, Genève, Droz, 1965 et J. Dufournet, Adam de la Halle à la recherche de lui-même ou le jeu dramatique de la Feuillée, Paris, SEDES, 1974, p. 71-100.

${ }^{44}$ Prise d'Orange, v. 666-667.

${ }^{45}$ On peut en effet comparer ces vers, qui dissocient les deux couleurs avant de les amplifier, à la subtile carnation d'Énide (Érec et Énide, éd. M. Roques, Paris, Champion, 1990, v. $427-$ 432), de Soredamour (Cligès, éd. A. Micha, Paris, Champion, 1982, v. 810-812) ou de Blanchefleur dans le Conte du Graal. La pureté de son teint se marie harmonieusement avec l'éclat du vermeil : «Et miauz avenoit an son vis / Li vermauz sor le blanc assis / Que li sinoples sor l'arjant» (v. 1823-1825). S'inspirant du même topos, les auteurs épiques cités par C. Lachet, op. cit., p. 87, n'unissent pas les deux teintes avec autant de grâce que Chrétien de Troyes. Ils ne les séparent toutefois pas aussi nettement que l'auteur de la Prise d'Orange: "Et fu blanche et vermeille, a point encoloree», Buevon de Conmarchis, éd. A. Henry, Euvres d'Adenet le Roi, t. II, Bruges, 1953, v. 2098 ; «Madame est fleur de lis et rose de saison », ibid., v. 3335 ; «Le vis blanc et traitis, la fache couloree », Doon de Mayence, éd. A. Pey, Paris, 1858, v. 7909 ; «La car out tendre et blanche comme flor enn esté, / La fache vermeillete conme rose de pré », Fierabras, éd. M. Le Person, Paris, Champion, 2003,
} v. 2109-2110. 
rouge, et mise en exergue par les diérèses: Rosiane évoquant la couleur rose et Rubiant le rubis, une gemme rouge.

Quant à la rencontre amoureuse, habituellement source d'émotion et de pathos, elle est également traitée avec humour, le style épique hyperbolique permettant de caricaturer les émotions du héros. À la vue d'Orable, la réaction de Guillaume s'avère en effet très violente, et l'expression de son désir curieusement proche des extrêmes manifestations de colère ${ }^{46}:$ " Voit la Guillelmes, tot li mua le sanc $\aleph^{47}$. Succédant au cliché courtois de la beauté féminine, ce décasyllabe appuyé par la parataxe asyndétique mime l'impétuosité et l'immédiateté de la réaction dans un style épique ${ }^{48}$, avant de provoquer un inévitable comique de contraste. À la délicatesse et au raffinement féminins succède sans transition une réaction brutale, formulée de manière stéréotypée. L'absence d'affinités entre ces deux univers est telle que le héros au sang bouillonnant tente d'adopter des manières courtoises, provoquant, dans une chute appuyée par la césure épique, un nouveau contraste amusant avec le motif épique des salutations : «Il la salüe bel et cortoisement $»^{49}$. Ses aspirations transparaissent dans la réduplication des adverbes en gradation rythmique, mais restent muettes si l'on considère la teneur bien peu courtoise de ses propos: «Cil Dex vos saut, en cui somes creant! $»^{50}$ En quatre vers, l'auteur a caricaturé et confronté alternativement les styles courtois et épique, suggérant le désarroi d'un héros amoureux, sensible aux charmes d'Orable, mais incapable, malgré ses efforts, de prononcer les termes délicats qu'il lui destine en intention, et contraint de ruser pour sauver les apparences sans être parjure.

L'association aussi étroite de ces deux esthétiques prête à sourire, et la réécriture de la scène de rencontre, susceptible d'occasionner un échange verbal sentimental, voire des aveux, subit un traitement parodique, car Guillaume et Orable ne sont pas seuls. Si l'expression «Dejoste lui» accentue en début de vers la proximité d'Orable, ce rapprochement spatial n'engendre aucune intimité, le complément de lieu étant suivi par un pronom personnel au pluriel : «Dejoste lui les asist sor un banc $\aleph^{51}$. La description subséquente confirme alors l'embarras de Guillaume, qui laisse errer son regard sur le banc, alors même que le narrateur insiste sur la possibilité d'une requête d'amour: "Or pueent dire auques de lor talent $~^{52}$. La timidité de l'amant pourrait assimiler Fierebrace aux amants courtois si les vers 676 à 679 ne témoignaient d'un intérêt accru pour le cadre spatial, tandis qu'Orable, exclue de la conversation, fait à peine partie du décor jugé paradisiaque. La scène d'aveux tant attendue est ainsi escamotée; grâce à un habile transfert, elle est transfigurée en une amusante conversation entre l'oncle et le neveu, au cours de

${ }^{46}$ Cf. Charroi de Nimes, v. 1212-1213 ; Moniage Guillaume, éd. W. Cloetta, Paris, 19061911, I, v. 488 ; Beuve de Hantone, éd. A. Stimming, Halle, 1911, t. 1, v. 170, 1929.

${ }^{47}$ Prise d'Orange, v. 668.

${ }^{48}$ Ce vers présente une structure stéréotypée avec inversion du sujet : verbe de perception + $\mathrm{CRD}+$ sujet + réaction.

${ }^{49}$ Prise d'Orange, v. 669.

${ }^{50}$ Prise d'Orange, v. 670.

${ }^{51}$ Prise d'Orange, v. 673.

${ }^{52}$ Prise d'Orange, v. 675. 
laquelle les charmes d'Orange semblent éclipser ceux d'Orable. La reverdie est ainsi encore parodiée par dissémination.

La difficulté de Guillaume à déclarer sa flamme tout en dissimulant son identité aurait pu justifier aisément le retard des aveux. Or l'auteur rejette cette solution de facilité et parodie le topos des aveux par inversion et éclatement. Le premier renversement est assez net: alors que Guillaume a risqué sa vie pour conquérir Orable, fasciné et intimidé, il n'ose finalement pas lui révéler ses sentiments. C'est donc Orable qui, à plusieurs reprises, lui suggère de plus en plus explicitement qu'elle l'aime : d'abord par un éloge discret ${ }^{53}$, puis en dotant le héros des armes de son époux ${ }^{54}$, enfin en proposant un chantage au mariage ${ }^{55}$. À l'instar des princesses sarrasines ${ }^{56}$, elle prend donc des initiatives et fait preuve d'audace, dérogeant de fait aux préceptes courtois malgré sa mesure. Quant à Guillaume, il parle à peine et n'adresse pas le moindre compliment à la reine ; il incarnerait le topos lyrique de l'amant anéanti par la timidité s'il ne se lançait, en contrepartie, dans un autoportrait dithyrambique ${ }^{57}$. Appuyé par un comique de situation et un subtil comique de caractère, le transfert parodique prête à sourire. Il s'amplifie lorsqu'en prison, Guillaume fait preuve de mauvaise foi et accuse Orable de trahison, alors qu'elle vient de leur sauver la vie. Remettant en cause à tort la loyauté et la confiance qui doit unir des amants, ses aveugles récriminations renversent les préceptes courtois et introduisent la discorde au sein d'un couple encore virtuel! Victimes d'un renversement parodique, les tendres aveux tant attendus se métamorphosent en d'amers reproches, et dans un subtil transfert dupliqué, les sarcasmes ironiques de Guïelin poursuivent cette réécriture distanciée dans les laisses XLIII à XLV. Promettant à Orable d'être son «hom» si elle les délivre, le neveu se substitue en effet à son oncle pour mieux dénoncer sa prostration et contrefaire l'amant courtois avouant son amour à demi-mot, le terme «hom » pouvant être compris dans son sens féodal ou dans son sens courtois. Le vocabulaire laudatif qui encadre la requête en chiasme, le déferlement d'hyperboles et les structures binaires rendent compte d'un discours caricatural autant destiné à faire réagir Guillaume qu'à obtenir leur libération :

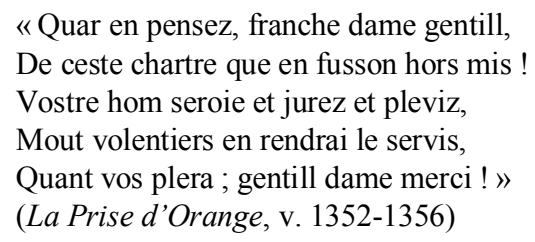

Simultanément, Guïelin dégrade le motif courtois élevé de l'amant soumis à sa dame et le tourne en dérision par un jeu héroï-comique, sa déclaration bassement

\footnotetext{
${ }^{53}$ « Par Mahomet, il doit bien tenir marche; / Liee est la dame en cui est son coraige », Prise d'Orange, v. 732-733.

${ }^{54}$ Prise d'Orange, v. 941-956.

${ }^{55}$ Prise d'Orange, v. 1374-1378.

${ }^{56}$ Voir l'analyse de C. Lachet, op. cit., p. 79-129.

${ }^{57}$ Prise d'Orange, v. 725-730.
} 
intéressée consistant à vendre sa soumission, dont la valeur est paradoxalement estimée à celle de la liberté. Dénonçant l'aliénation courtoise, la parodie procède par inversion: Guielin n'est pas amoureux d'Orable, et loin d'offrir gracieusement une obédience absolue, il demande d'abord une faveur, être libéré : «Si estions mis hors de ceste chartre / Vostre hom seroie a trestoz mon aaige $»^{58}$. Ainsi inspire-t-il indirectement Orable, qui reprend ce raisonnement et propose un marché à Guillaume : la libération contre le mariage et le baptême. Au terme d'une série de transferts ludiques, la Sarrasine amoureuse est ainsi contrainte d'assumer son rôle et de prendre des initiatives, alors que Fierebrace est plus désarmé que jamais.

La réécriture distanciée de la reverdie traverse ainsi toute l'œuvre pour s'achever à l'aube du dénouement, son emploi en clôture narrative renversant ses traditionnelles fonctions d'ouverture et de relance. Transférant sur Bertrand les vers 43 à 52 initialement consacrés à Guillaume dans un écho épique destiné à clore la chanson et à annoncer la victoire des chrétiens, la deuxième partie de la laisse LVII engendre en outre un comique de répétition qu'amplifient de subtils décalages, la nouvelle attribution de la conquête de Nîmes à Bertrand et une nostalgie tout épique: le regard perdu dans cette reverdie, Bertrand souffre de l'absence de son oncle et de son frère, alors que Guillaume, en harmonie avec le paysage, regrettait la vie de plaisir «que il soloit en France demener »!

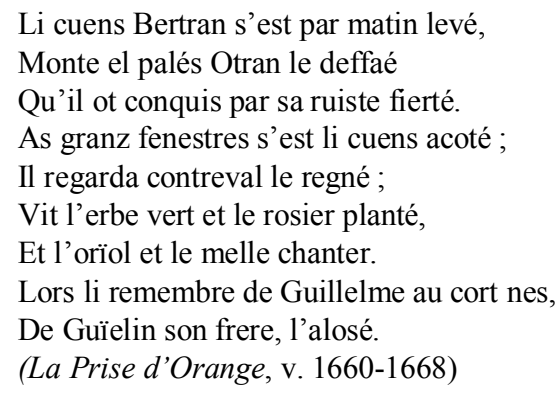

Dans ce contexte lyrique et courtois amplifié par la reprise de la laisse III, les préoccupations épiques de Bertrand paraissent déplacées et provoquent le sourire: ne pense-t-il pas à Guillaume et à Guïelin dans la posture où Fierebrace rêvait de demoiselles susceptibles d'offrir des divertissements courtois, voire sensuels? Dans un texte ludique qui se referme sur lui-même en se réécrivant, le transfert parodique émousse le pathos de la situation et ménage une transition humoristique vers un dénouement à peine plus canonique.

Omniprésent, le modèle lyrique de la reverdie émaille ainsi la chanson de geste de ses teintes chatoyantes, introduisant ruptures et jeux déceptifs. $\mathrm{Si}$ «la parodie de l'épopée relève essentiellement de la présence de la courtoisie», si «les écarts parodiques sont dus à l'introduction de traits et des termes courtois dans un domaine épique figé $»^{59}$, ce motif lyrique n'échappe pas à une réécriture distanciée et

\footnotetext{
${ }^{58}$ Prise d'Orange, v. 1372-1373.

${ }^{59}$ C. Lachet, op. cit., p. 221.
} 
humoristique, procédant par éclatement, inversion, transfert et réduplication, et s'enrichissant de procédés comiques fondés essentiellement sur les contrastes et les situations. Par l'intrusion régulière et cohérente d'éléments exogènes lyriques également parodiés, le poète a donc réussi à donner le jour à une œuvre kaléidoscopique riche et complexe. Assurément, le jeu parodique est une tentation à laquelle ce trouvère n'a guère su résister!

Lydie Louison Université Jean Moulin-Lyon 3 